Ophthalmologe 2011 · 108:102-103

DOI 10.1007/s00347-010-2288-3

Online publiziert: 27. Januar 2011

(c) Springer-Verlag 2011

\author{
L-O. Hattenbach \\ Augenklinik des Klinikums Ludwigshafen
}

\title{
Diagnostik bei venösen retinalen Gefäßverschlüssen
}

Der Beitrag von Mirshahi et al. berichtet über aktuelle Aspekte der OCT-basierten Diagnostik bei venösen retinalen Gefäßverschlüssen ebenso wie über klassische bildgebende Verfahren und diagnostische Maßnahmen zur Identifizierung ophthalmologischer Risikofaktoren, die auf der „Fahndungsliste“ für venöse retinale Gefäßverschlüsse nicht fehlen dürfen. Mittlerweile ist eine Reihe von Arbeiten entstanden, die zeigen, dass die typischerweise mit einem venösen retinalen Gefäßverschluss einhergehenden und lange Zeit relativ einheitlich definierten Veränderungen wie das Makulaödem durchaus eine erhebliche Variabilität aufweisen und sich von ähnlichen, durch andere Krankheitsbilder bedingten Pathologien signifikant unterscheiden.

\section{จ Die Weiterentwicklung der OCT bietet bisher ungekannte Differenzierungsmöglichkeiten}

Zudem wurde erkannt, dass das Muster der mit einem venösen Netzhautgefäßverschluss einhergehenden Veränderungen offenbar Einfluss auf Verlauf und Prognose hat. So zeigte sich bei Patienten mit Zentralvenenverschluss eine statistisch signifikante Korrelation zwischen initialer Netzhautdicke und Visus. Bei Patienten mit Venenastverschluss waren intraretinale zystoide Räume mit einem Durchmesser $>600 \mu \mathrm{m}$ assoziiert mit einem längeren Krankheitsverlauf und einem schlechteren Ansprechen nach intravitrealer Injektionstherapie mit Anti-VEGF-Wirkstoffen, während bei Patienten mit Zentralvenenverschluss für das Vorliegen von subretinaler Flüssigkeit oder den Durchmesser der intraretinalen zystoiden Räume kein signifikanter Einfluss beobachtet wurde. Andere Autoren konnten mittels „Spectral-domain-OCT“ demonstrieren, dass man bei einem Makulaödem nach venösen Verschlüssen der Netzhaut und im Rahmen einer diabetischen Makulopathie häufiger zystoide Veränderungen findet als bei durch andere Ursachen bedingten Ödemen.

Fortschritte der Medizin haben in den vergangenen Jahren auf dem Gebiet der retinalen Gefäßverschlusserkrankungen auch zu einer weiteren „stillen“ Revolution geführt. Galt es bis vor wenigen Jahren noch als heftig umstritten, inwieweit thrombophile Gerinnungsstörungen bei der Entstehung venöser retinaler Gefäßverschlüsse überhaupt eine Rolle spielen, belegt mittlerweile eine Reihe von Arbeiten einen eindeutigen Zusammenhang. Eine wichtige Voraussetzung für diese Erkenntnis war die Entdeckung der bis heute häufigsten bekannten Gerinnungsstörung, der Resistenz gegen aktiviertes Protein C (APC-Resistenz), die Anfang der 1990er-Jahre von Dahlbäck et al. erstmals beschrieben wurde und damit die Wahrscheinlichkeit für den Nachweis von Gerinnungsdefekten bei Risikopatienten generell erhöhte.

Der Beitrag von Kuhli-Hattenbach et al. zeigt jedoch, dass noch eine andere methodische Voraussetzung für eine erfolgreiche ophthalmologische Thrombophiliediagnostik unabdingbar ist: Die Festlegung von Kriterien für eine geeignete Patientenselektion. Lange Zeit wurden Untersuchungen zur Thrombophi- 
lie bei Netzhautgefäßverschlüssen nur anhand zufällig zusammengesetzter und meist kleiner Patientengruppen ohne Vergleichskollektiv durchgeführt und dabei teilweise sogar jene Fälle ausgeschlossen, die eine auffällige Eigen- oder Familienanamnese hinsichtlich Thrombembolien großer Gefäße aufwiesen. Venöse retinale Gefäßverschlüsse sind jedoch eine Erkrankung des höheren Lebensalters und treten daher meist in Assoziation mit kardiovaskulären Grunderkrankungen wie der arteriellen Hypertonie auf. Bei jüngeren Patienten mit venösem retinalem Gefäßverschluss - immerhin etwa jeder Zehnte ist zum Zeitpunkt des Erkrankungsbeginns 50 Jahre oder jünger - trifft dies allerdings häufig nicht zu. Stattdessen findet sich gerade in dieser Altersgruppe anamnestisch nicht selten eine familiäre Häufung oder ein vorangegangenes thrombembolisches Ereignis als Hinweis auf eine entsprechende Prädisposition. So konnten in größeren Fallserien anhand von Subgruppenanalysen ein Alter $\leq 45$ Jahre zum Zeitpunkt des ersten thrombembolischen Ereignisses, das Fehlen kardiovaskulärer Risikofaktoren unabhängig vom Alter sowie bei Patienten $\leq 60$ Jahre eine auffällige Familienanamnese hinsichtlich thrombembolischer Ereignisse als wesentliche Kriterien für den Verdacht auf das Vorliegen einer thrombophilen Gerinnungsstörung identifiziert werden.

Und noch ein weitere Erkenntnis wird deutlich: Auch bei venösen Netzhautgefäßverschlüssen führen offenbar bestimmte Gerinnungsdefekte gefäßbettspezifisch zu einer Erhöhung des Thromboserisikos. So weisen verfügbare Daten eindeutig darauf hin, dass der FaktorXII-Mangel, eine in der Normalbevölkerung sehr seltene Gerinnungsstörung, für die eher kein kausaler Zusammenhang mit systemischen Thrombosen großer Gefäße angenommen wird, als spezifischer thrombophiler Risikofaktor für Perfusionsstörungen in mikrovaskulären Stromgebieten betrachtet werden kann.

Venöse retinale Gefäßverschlüsse stellen ein insgesamt sehr inhomogenes Krankheitsbild dar, das hinsichtlich der Prognose von zahlreichen Faktoren beeinflusst wird und dessen Entstehung mit unterschiedlichen okulären und sys- temischen Grunderkrankungen oder Risikofaktoren assoziiert sein kann. Eine adäquate, für den individuellen Patienten geeignete Versorgung kann daher nur durch eine gezielte, an evidenzbasierten Kriterien orientierte Diagnostik und detaillierte Interpretation der zur Verfügung stehenden Befunde erreicht werden.

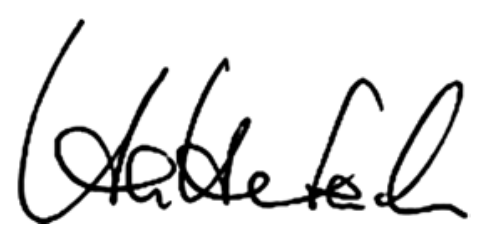

Lars-Olof Hattenbach

\section{Korrespondenzadresse \\ Prof. Dr. L-O. Hattenbach}

Augenklinik des Klinikums Ludwigshafen Bremserstr. 79, 67063 Ludwigshafen Hattenbach.LO@klilu.de

\section{Ausschreibung von Preisen und Forschungsförderungen der DOG in 2011}

\section{Glaukomforschungspreis}

Für das Jahr 2011 schreibt die DOG den Glaukomforschungspreis aus zur Anerkennung für herausragende Arbeiten auf dem Gebiet der experimentellen und klinischen Glaukomatologie. Der Preis wird gestiftet von der Firma Santen $\mathrm{GmbH}$, die Dotierung beträgt 5.000 Euro.

Bewerben können sich Nachwuchswissenschaftler/-innen an Universitätskliniken oder Universitätsinstituten sowie wissenschaftlich orientierte, praktizierende Augenärzte/ Augenärztinnen mit besonderem Interesse am Glaukom, welche das 40. Lebensjahr noch nicht überschritten haben.

Bewerbungsarbeiten sind in fünffacher Ausfertigung einfach oder in Form eines Sonderdruckes zusammen mit einem Curriculum vitae der Bewerberin/des Bewerbers sowie einer Aufstellung und Beschreibung von bereits erhaltenen Förderungen und Auszeichnungen einzureichen bei der Geschäftsstelle der DOG, z.Hd. des Schriftführers, Platenstraße 1, 80336 München. Bewerbungsarbeiten können veröffentlicht oder unveröffentlicht sein. Veröffentlichte wissenschaftliche Originalarbeiten auf dem Gebiet der Glaukomatologie sollten nicht älter als 2 Jahre sein. Einsendeschluss für Bewerbungen ist der 1. Mai 2011.

Die Bewerbungsarbeit wird in deutscher oder englischer Sprache akzeptiert. 